\title{
Patrones de desplazamiento al centro educativo en adolescentes de Huesca
}

\section{Commuting patterns to school in adolescents from Huesca}

\author{
Laura Simón-Montañés ${ }^{1}$; Alberto Aibar ${ }^{2}$; Ángel Abós ${ }^{1}$; \\ Luis García-González ${ }^{1}$ y Javier Sevil-Serrano ${ }^{1}$ \\ ${ }^{1}$ Facultad de Ciencias de la Salud y del Deporte, Zaragoza, España \\ ${ }^{2}$ Facultad de Ciencias Humanas y de la Educación, Zaragoza, España. \\ Contacto: laura.simon.mon@gmail.es
}

Este trabajo ha sido financiado por el Ministerio de Economía y Competitividad (MINECO; EDU2013-42048-R), el Gobierno de Aragón y el Fondo Social Europeo. 


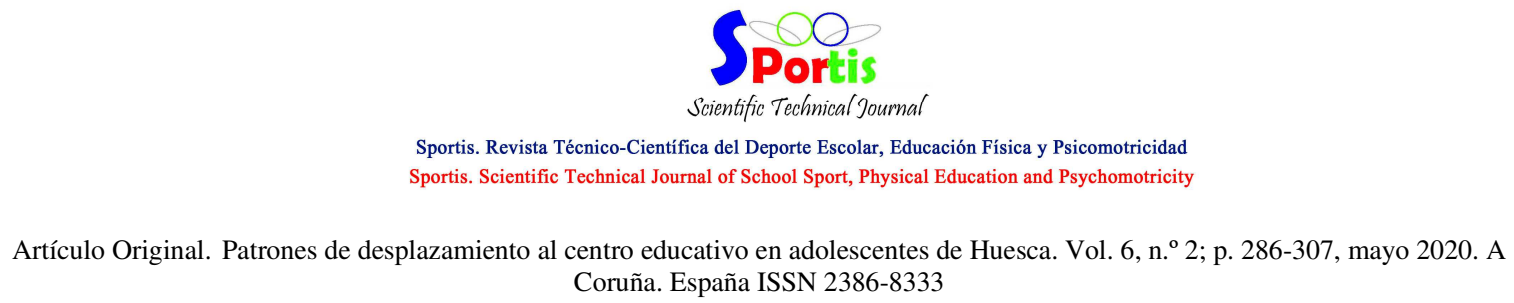

\title{
Resumen
}

El objetivo del estudio fue describir el tipo de desplazamiento utilizado por los adolescentes de Huesca para ir y volver del centro educativo, así como analizar su asociación con el género y el curso académico. Participaron 1.709 adolescentes (Medad=14.20; DT=1.56; 51.7\% chicas) de $1^{\circ}$ de Educación Secundaria Obligatoria (ESO) a $1^{\circ}$ de Bachillerato. Los estudiantes cumplimentaron un cuestionario sobre el modo habitual de desplazamiento en la ida y vuelta al instituto. Los resultados señalaron que un $76.4 \%$ de los adolescentes se desplazaba activamente (i.e., andando o en bicicleta) tanto en la ida como en la vuelta. De los adolescentes que se desplazaban activamente, un $78.5 \%$ en la ida y un $81.7 \%$ en la vuelta lo hacía caminando. En relación con el género, se encontró una asociación positiva entre ir andando al centro educativo y ser chica, así como entre ir en bicicleta y ser chico. En relación con el curso académico, desplazarse andando al centro educativo se asoció positivamente con $4^{\circ}$ de ESO y negativamente con $1^{\circ}$ de Bachillerato. Asimismo, desplazarse en bicicleta se asoció positivamente con $1^{\circ}$ de Bachillerato y negativamente con $3^{\circ}$ de ESO. Aunque la mayoría de los adolescentes se desplaza activamente al instituto, parece necesario promover programas escolares que incentiven el desplazamiento activo de los jóvenes dada las características favorables de movilidad de la ciudad de Huesca. De igual modo, parece necesaria la promoción de la bicicleta como modo de desplazamiento al instituto, especialmente en las chicas y en los estudiantes de ESO.

\section{Palabras clave}

Desplazamiento activo; bicicleta; andar; género; curso académico.

\begin{abstract}
The aim of this study was to describe the usual mode of commuting to and from school in adolescents of Huesca and its association with gender and academic year. A total of 1,709 adolescents (Mage $=14.20 ; D T=1.56 ; 51.7 \%$ girls) from $1^{\text {st }}$-year of middle school [Spanish acronym, ESO] to $1^{\text {st }}$-year high school) participated in this study. A questionnaire of the usual mode of commuting to and from school was self-reported by students. Results showed that $76.4 \%$ of adolescents commuted actively (i.e., walking or cycling) to and from school. Of the adolescents who commute actively to and from school, $78.5 \%$ to school and $81.7 \%$ from school walked. Regarding gender, a positive association was found between walking to and from school and being a girl, as well as between cycling and being a boy. Regarding the grade level, walking to and from school was positively associated with adolescents in $4^{\text {th }}$ of middle school and negatively with $1^{\text {st }}$-year high school. On the other hand, travelling by bicycle both routes was positively associated with $1^{\text {st }}$-year of high school and negatively with $3^{\text {rd }}$ year of middle school. Although the majority of adolescents from Huesca commuted actively to and from school, it seems that active school transport interventions are necessary, given the favorable characteristics of the city of Huesca. Similarly, it seems necessary to promote cycling as a means of transport to and from school, especially among girls and secondary education students.
\end{abstract}

Para citar este artículo utilice la siguiente referencia: Simón-Montañés, L.; Aibar, A.; Abós, A.; García-González, L.; Sevil-Serrano, J. (2020).

Patrones de desplazamiento al centro educativo en adolescentes de Huesca. Sportis Sci J, 6 (2), 286-307. DOI: https://doi.org/10.17979/sportis.2020.6.2.5975 


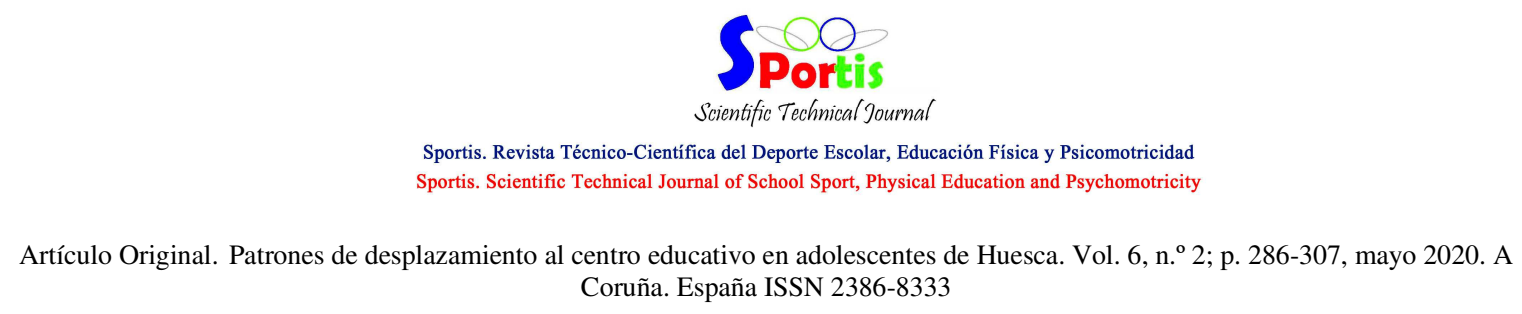

\section{Keywords}

Active commuting; cycling; walking; gender; academic grade.

\section{Introducción}

La práctica regular de actividad física (AF) conlleva una serie de beneficios físicos, psicológicos y sociales (Poitras et al., 2016). Sin embargo, la mayoría de los niños y adolescentes no cumple las recomendaciones de AF (i.e., al menos 60 minutos/día de actividad física moderada a vigorosa [AFMV]; World Health Organization, 2010), tanto en España (Roman-Viñas, Zazo, Martínez-Martínez, Aznar-Laín, y Serra-Majem, 2018) como a nivel internacional (Guthold, Stevens, Riley, y Bull, 2020).

Una de las medidas para incrementar los bajos niveles de AF de los jóvenes es la promoción del desplazamiento activo al centro educativo. El desplazamiento activo supone trasladarse al centro escolar en bicicleta o andando. Por el contrario, desplazarse de forma pasiva consiste en el uso de un medio de transporte motorizado (e.g., autobús, coche, etc.) (Herrador-Colmenero, Escabias, Ortega, McDonald, y Chillón, 2019; Larouche, Saunders, John, Colley, y Tremblay, 2014). Diferentes revisiones sistemáticas han demostrado que desplazarse de forma activa al centro escolar podría incrementar los niveles de AF (Larouche et al., 2014) y mejorar la condición física, especialmente cuando los jóvenes se desplazan en bicicleta (Larouche et al., 2014). Asimismo, desplazarse caminando al colegio favorece el bienestar y las relaciones sociales entre los estudiantes (Waygood, Friman, Olsson, y Taniguchi, 2017) y puede producir mejoras en el rendimiento académico (Stea y Torstveit, 2014). A nivel medio-ambiental, el desplazamiento activo permite disminuir la contaminación atmosférica y acústica derivada del desplazamiento con vehículos motorizados (Gössling, Choi, Dekker, y Metzler, 2019). Por tanto, el desplazamiento activo a la escuela (i.e., andando o en bicicleta) podría incrementar los niveles de AF de la población a la vez que se reducen los niveles de contaminación y, en consecuencia, mejoraría la sostenibilidad de las ciudades. Concretamente, fomentar la movilidad en bicicleta desde las instituciones educativas ayuda a conseguir 12 de los 17 Objetivos de Desarrollo Sostenible de la Agenda 2030 (Organización de las Naciones Unidas 2015; Red de ciudades por la bicicleta, 2019).

Para citar este artículo utilice la siguiente referencia: Simón-Montañés, L.; Aibar, A.; Abós, A.; García-González, L.; Sevil-Serrano, J. (2020). 


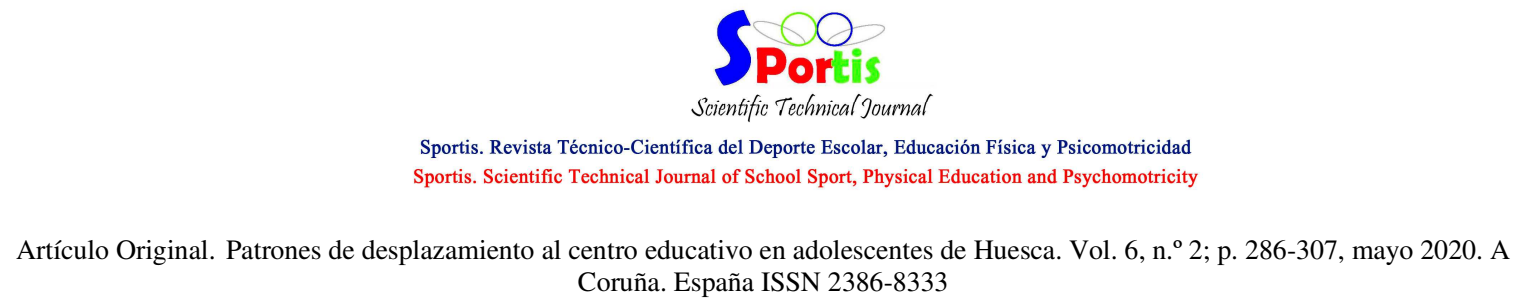

A pesar de los múltiples beneficios asociados al desplazamiento activo al centro escolar, en los últimos años numerosos estudios nacionales (Chillón et al., 2013) e internacionales (Booth, Rowlands, y Dollman, 2019) han mostrado un descenso en los patrones de desplazamiento activo en jóvenes. Por ejemplo, un estudio longitudinal de seis años de duración llevado a cabo en adolescentes residentes en España, evidenció un descenso de los patrones de transporte activo de un $61 \%$ a un $48 \%$ en chicas (Chillón et al., 2013). A nivel nacional, diferentes estudios nos muestran como aproximadamente entre un 46\% (SanzArazuri, Ponde-de-León, y Fraguela-Vale, 2017) y un 71\% (Aparicio-Ugarriza et al., 2020) de los adolescentes españoles se desplazan activamente al centro escolar, siendo menos de un $2 \%$ los que lo hacen a través de la bicicleta. Concretamente, en Aragón, una reciente investigación reveló que hasta un $68 \%$ de los adolescentes se desplazaba de forma activa al centro educativo (Abarca, Gallardo, Generelo, Julián, y Zaragoza, 2019). Las diferencias encontradas en los patrones de desplazamiento activo en escolares podrían deberse al contexto de referencia en el que se realizaron dichos estudios, por lo que es necesario seguir investigando este comportamiento saludable en diferentes ciudades españolas con el fin de analizar correctamente la prevalencia de este comportamiento a nivel nacional.

Por otro lado, una revisión sistemática señaló que la mayoría de los estudios previos únicamente han evaluado los patrones de desplazamiento en la ida al centro educativo (Herrador-Colmenero, Pérez-García, Ruiz-Ruiz, y Chillón, 2014). Por tanto, parece necesario seguir profundizando en el estudio del trayecto de vuelta al domicilio. En el limitado número de estudios existente, tanto a nivel nacional como internacional, se ha visto que el modo de transporte de los adolescentes al centro educativo es diferente entre la ida y la vuelta, siendo más alto el porcentaje de jóvenes que se desplazan caminando en la vuelta (HerradorColmenero et al., 2019; Larsen, Gilliland, y Hess 2012; Ruiz-Ariza, García, Latorre, y Martínez-López, 2015). Por ejemplo, en un estudio reciente llevado a cabo por Herrador et al. (2019) en adolescentes españoles, el desplazamiento andando fue significativamente mayor en la vuelta que en la ida (57.25\% vs. $54.84 \%)$, mientras que el desplazamiento en coche fue mayor en la ida que en la vuelta $(22.61 \%$ vs. $19.41 \%)$.

Para citar este artículo utilice la siguiente referencia: Simón-Montañés, L.; Aibar, A.; Abós, A.; García-González, L.; Sevil-Serrano, J. (2020). Patrones de desplazamiento al centro educativo en adolescentes de Huesca. Sportis Sci J, 6 (2), 286-307. DOI: https://doi.org/10.17979/sportis.2020.6.2.5975 


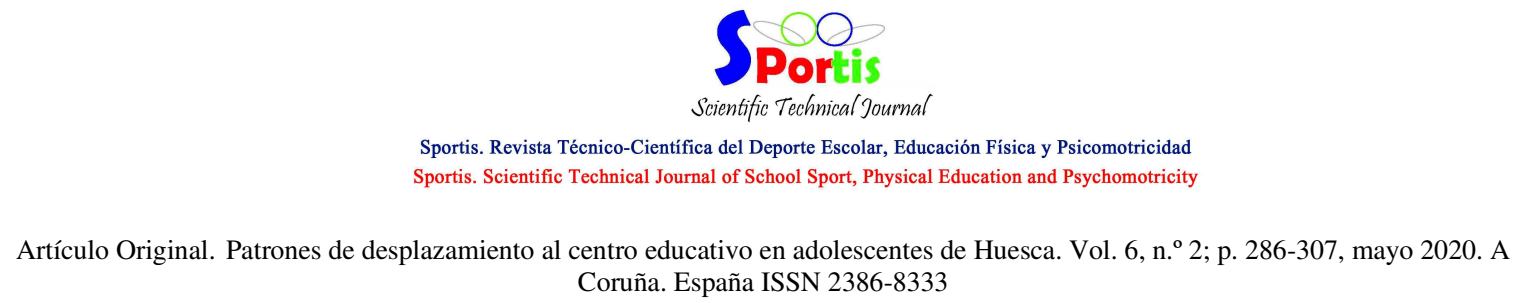

En relación con el modo de desplazamiento de los jóvenes y las posibles diferencias en función del género, no existen unos resultados concluyentes en la literatura científica. Un estudio que analizó los patrones de desplazamiento en más de 100.000 adolescentes de 27 países asiáticos reveló diferencias significativas en función del género en 9 de dichos países. Concretamente, en siete de los países se desplazaron más activamente los chicos respecto a las chicas, mientras que en dos de los países restantes ocurría lo contrario (Uddin, Mandic, y Khan, 2019). En otra revisión sistemática se observó que los chicos se desplazaban más en bicicleta, mientras que las chicas lo hacían en mayor medida caminando (Rojas y Wong, 2017). Sin embargo, en una reciente revisión sistemática no se encontraron diferencias en el desplazamiento activo al centro escolar en función del género tanto en la infancia como en la adolescencia (Kemp, Cliff, Chong, y Parrish, 2019). A nivel nacional, algunos estudios han señalado que las chicas tienen patrones de desplazamiento más activos que los chicos en todos los rangos de edad examinados (i.e., 13 a 18 años) (Chillón et al., 2009), mientras que en otros estudios no existían diferencias entre ambos géneros (Martínez-Gómez et al., 2011; Ruiz-Ariza et al., 2015; Sanz-Arazuri et al., 2017). Cabe destacar que en un estudio las chicas se desplazaron ligeramente más en bicicleta para ir al centro escolar que los chicos (SanzArazuri et al., 2017), mientras que en otros tres estudios no existieron casi diferencias (Chillon et al., 2009; Chillón et al., 2013; Martínez-Gómez et al., 2011). La gran variabilidad de resultados encontrados hace necesario seguir estudiando las diferencias de desplazamiento activo en los adolescentes en función del género.

Respecto al curso académico o la edad, tampoco existen resultados unívocos en la literatura científica. Por ejemplo, un estudio transcultural en 27 países asiáticos determinó que los adolescentes más mayores (i.e., $1^{\circ}$ de Bachillerato; 16-17 años), se desplazaban a través de modos de desplazamiento más activos que los más jóvenes (i.e., $2^{\circ}$ y $3^{\circ}$ de Educación Secundaria Obligatoria [ESO]; 13-15 años) en seis de los países analizados. Sin embargo, en otros siete países ocurrió lo contrario, siendo los adolescentes más jóvenes los que más se desplazaban activamente (Uddin et al., 2019). Otro estudio desarrollado con jóvenes estadounidenses reveló que los estudiantes de 12 a 14 años (i.e., $1^{\circ}$ y $2^{\circ}$ de ESO; 11.7\%) se desplazaron más en bicicleta y caminando que sus homólogos de 15 a 17 años (i.e., $4^{\text {o }}$ de ESO y $1^{\mathrm{o}}$ de Bachillerato; 8.8\%) (Kontou, McDonald, Brookshire, Pullen-Seufert, y LaJeunesse

Para citar este artículo utilice la siguiente referencia: Simón-Montañés, L.; Aibar, A.; Abós, A.; García-González, L.; Sevil-Serrano, J. (2020).

Patrones de desplazamiento al centro educativo en adolescentes de Huesca. Sportis Sci J, 6 (2), 286-307. DOI: https://doi.org/10.17979/sportis.2020.6.2.5975 


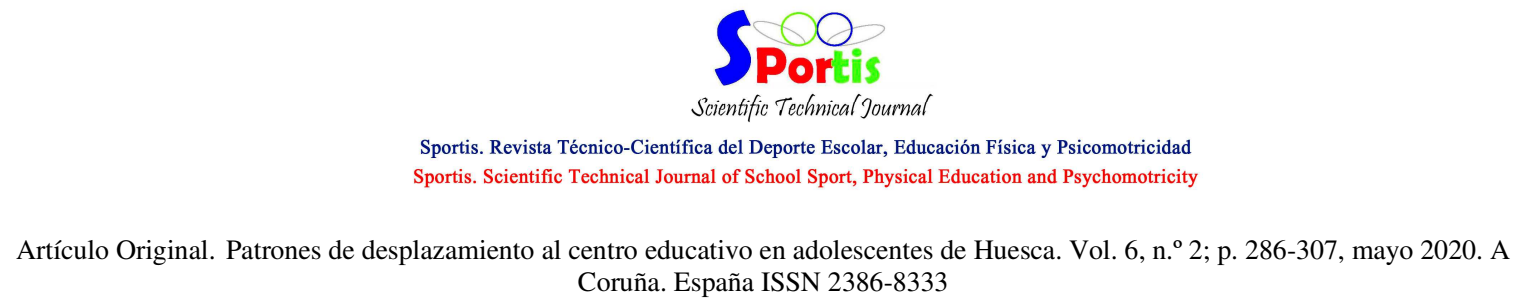

2019). A nivel nacional, algunos estudios han señalado que los niños de Educación Primaria se desplazan más activamente que los adolescentes de ESO (e.g., Herrador et al., 2019; Rodríguez-López, Villa-González, Herrador-Colmenero, Ortega, y Chillón 2016). Asimismo, el estudio llevado a cabo por Chillón et al. (2009) determinó que los jóvenes de la primera etapa de la adolescencia (i.e., 13 a 15 años) se desplazaron más activamente que los jóvenes de 17 a 18 años. No obstante, dada la limitación en el número de estudios, parece necesario seguir investigando cómo evolucionan los patrones de desplazamiento a lo largo de la etapa adolescente para adecuar los programas escolares de promoción del desplazamiento activo a cada curso académico.

Por tanto, dado que los patrones de desplazamiento de los adolescentes españoles al centro escolar podrían variar en función de las características de la ciudad examinada, parecen necesarios más estudios en diferentes regiones españolas que profundicen en este ámbito de estudio. Asimismo, teniendo en cuenta los resultados mixtos, tanto en función del género como del curso académico, parecen necesarios más estudios que arrojen luz sobre la influencia de estos dos factores en el desplazamiento de los adolescentes. Consecuentemente, el objetivo del estudio fue describir el tipo de desplazamiento utilizado para ir y volver al centro educativo en una muestra de adolescentes de la ciudad de Huesca, así como analizar la asociación entre el género y el curso académico de los estudiantes y el tipo de transporte utilizado en el desplazamiento habitual de ida, el de vuelta y el de ida y vuelta.

\section{Material y método}

\section{Diseño y participantes}

Se realizó un estudio descriptivo transversal en el que participaron un total de 1.709 estudiantes (Medad $=14.20 ; D T=1.56 ; 51.7 \%$ chicas) con edades comprendidas entre 12 y 17 años. Dichos estudiantes pertenecían a seis de los ocho centros educativos públicos y concertados de la ciudad de Huesca. Concretamente, participaron 304 estudiantes de $1^{\circ}$ de ESO (17.80\%), 458 de $2^{\circ}$ de ESO (26.80\%), 226 de $3^{\circ}$ de ESO (13.20\%), 436 de $4^{\circ}$ de ESO (25.50\%) y 285 de $1^{\circ}$ de Bachillerato (16.70\%).

Para citar este artículo utilice la siguiente referencia: Simón-Montañés, L.; Aibar, A.; Abós, A.; García-González, L.; Sevil-Serrano, J. (2020). Patrones de desplazamiento al centro educativo en adolescentes de Huesca. Sportis Sci J, 6 (2), 286-307. DOI: https://doi.org/10.17979/sportis.2020.6.2.5975 


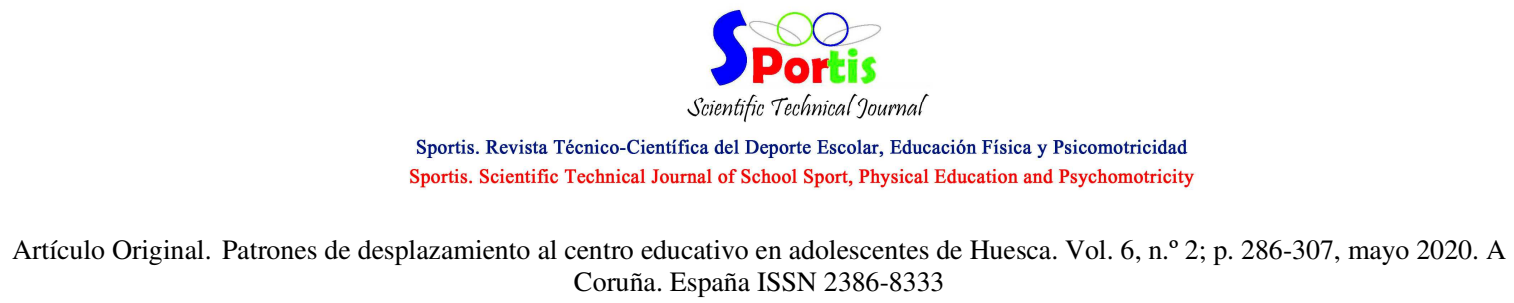

Instrumento

Para evaluar el modo habitual de desplazamiento habitual para ir y volver al centro educativo se utilizaron dos preguntas específicas de un cuestionario validado (Chillón et al., 2017), siguiendo los hallazgos sugeridos por una revisión sistemática previa (HerradorColmenero et al., 2014). Las dos primeras preguntas tenían las siguientes cinco opciones de respuesta: "pie", "bicicleta", "coche”, "motocicleta" y "autobús". Dadas las posibles opciones de respuesta, en algunos análisis se categorizó el desplazamiento en: activo (i.e., andando y en bicicleta) y pasivo (i.e., coche, autobús y motocicleta). Estas preguntas han sido utilizadas en otros estudios previos en población adolescente (Chillón, et al., 2009; Martínez-Gómez et al., 2011).

\section{Procedimiento}

En primer lugar, el equipo investigador contactó con los diferentes centros educativos con el objeto de explicarles el objetivo del estudio y solicitarles su participación. Seguidamente, se mandó una autorización por escrito a los padres o tutores legales del alumnado para obtener el consentimiento voluntario de participación. Los estudiantes tardaron entre 5 y 10 minutos en cumplimentar el cuestionario.

\section{Análisis de datos}

En primer lugar, se calcularon los estadísticos descriptivos (frecuencia y porcentaje) en el modo de desplazamiento de ida, vuelta, e ida y vuelta. Cabe destacar que para calcular los patrones de desplazamiento en la ida y vuelta al centro educativo se tuvieron en cuenta ambos trayectos. Posteriormente, para examinar el grado de asociación entre el el tipo de desplazamiento utilizado para ir y volver del centro educativo y el género y el curso académico se realizó la prueba Chi-cuadrado $\left(x^{2}\right)$. En dicho análisis, se reportó el coeficiente V de Cramer para identificar el tamaño del efecto de dichas asociaciones, considerándose bajo $(<.10)$, medio (>.10 y <.30) y alto (>.30) (Cohen, 1988). Todos los datos fueron analizados a través del software estadístico SPSS v.21.0.

\section{Resultados}

Para citar este artículo utilice la siguiente referencia: Simón-Montañés, L.; Aibar, A.; Abós, A.; García-González, L.; Sevil-Serrano, J. (2020). Patrones de desplazamiento al centro educativo en adolescentes de Huesca. Sportis Sci J, 6 (2), 286-307. DOI: https://doi.org/10.17979/sportis.2020.6.2.5975 
Artículo Original. Patrones de desplazamiento al centro educativo en adolescentes de Huesca. Vol. 6, n. ${ }^{\text {2 }}$; p. 286-307, mayo 2020. A Coruña. España ISSN 2386-8333

En la Tabla 1 puede apreciarse que un $76.5 \%$ se desplazó activamente tanto a la ida como a la vuelta del centro escolar, mientras que un $16.0 \%$ se desplazó pasivamente en ambos trayectos al centro educativo. Asimismo, un 5.3\% de los estudiantes se desplazó activamente en el camino de ida del centro educativo y pasivamente en el desplazamiento de vuelta, mientras que un $2.3 \%$ de los adolescentes se desplazó pasivamente en el camino de ida del centro educativo y activamente en el desplazamiento de vuelta. En relación con el género, se encontró una asociación positiva entre realizar ambos desplazamientos de forma activa y ser chica, así como entre desplazarse pasivamente en ambos trayectos y ser chico. En relación con el curso académico, realizar ambos desplazamientos activos se asoció positivamente con los adolescentes de $4^{\circ}$ de ESO y negativamente con los de $1^{\circ}$ de Bachillerato. Por otro lado, desplazarse activamente en la ida y pasivamente en la vuelta se asoció positivamente con los adolescentes de $1^{\circ}$ y $3^{\circ}$ de ESO y negativamente con los de $2^{\circ}$ de ESO. Finalmente, realizar ambos desplazamientos pasivos se asoció positivamente con los adolescentes de $1^{\mathrm{o}}$ de Bachillerato y negativamente con los de $4^{\circ}$ de ESO (ver Tabla 1).

Tabla 1. Patrones de desplazamiento en la ida y vuelta al centro educativo: diferencias en función del género y del curso académico.

\begin{tabular}{|c|c|c|c|c|c|c|}
\hline & $\begin{array}{c}\text { Ambos } \\
\text { desplazamientos } \\
\text { activos } \\
\mathrm{n}(\%) \\
\mathrm{rc} \\
\end{array}$ & $\begin{array}{l}\text { Ida activa } \\
\text { y vuelta } \\
\text { pasiva } \\
\mathrm{n}(\%) \\
\mathrm{rc} \\
\end{array}$ & $\begin{array}{c}\text { Ida pasiva } \mathbf{y} \\
\text { vuelta } \\
\text { activa } \\
\mathrm{n}(\%) \\
\mathrm{rc} \\
\end{array}$ & $\begin{array}{c}\text { Ambos } \\
\text { desplazamientos } \\
\text { pasivos } \\
\mathrm{n}(\%) \\
\mathrm{rc} \\
\end{array}$ & $x 2(g l)$ & V Cramer \\
\hline Total & $1305(76.4 \%)$ & $99(5.3 \%)$ & $40(2.3 \%)$ & $274(16.0 \%)$ & & \\
\hline \multicolumn{7}{|l|}{ Género } \\
\hline Chicos & $\begin{array}{c}594(73.0 \%) \\
-3.1\end{array}$ & $\begin{array}{c}37(4.5 \%) \\
-0.9\end{array}$ & $\begin{array}{c}14(1.7 \%) \\
-1.7\end{array}$ & $\begin{array}{c}169(20.8 \%) \\
4.8\end{array}$ & \multirow[t]{2}{*}{$25.542(3)^{* *}$} & \multirow[t]{2}{*}{.123} \\
\hline Chicas & $\begin{array}{c}692(79.4 \%) \\
3.1\end{array}$ & $\begin{array}{c}48(5.5 \%) \\
0.9\end{array}$ & $\begin{array}{c}26(3.0 \%) \\
1.7\end{array}$ & $\begin{array}{c}105(12.1 \%) \\
-4.8\end{array}$ & & \\
\hline \multicolumn{7}{|c|}{ Curso académico } \\
\hline $1^{\circ}$ de ESO & $\begin{array}{c}228(75.0 \%) \\
-0.6\end{array}$ & $\begin{array}{c}25(8.2 \%) \\
2.5\end{array}$ & $\begin{array}{c}11(3.6 \%) \\
1.6\end{array}$ & $\begin{array}{c}40(13.2 \%) \\
-1.5\end{array}$ & \multirow{5}{*}{$59.382(12)^{* *}$} & \multirow{5}{*}{.108} \\
\hline $2^{\circ}$ de ESO & $\begin{array}{c}359(78.4 \%) \\
1.2\end{array}$ & $\begin{array}{c}13(2.8 \%) \\
-2.7\end{array}$ & $\begin{array}{c}10(2.2 \%) \\
-0.3\end{array}$ & $\begin{array}{c}76(16.7 \%) \\
0.4\end{array}$ & & \\
\hline $3^{\circ}$ de ESO & $\begin{array}{c}174(77.0 \%) \\
0.2\end{array}$ & $\begin{array}{c}19(8.4 \%) \\
2.3\end{array}$ & $\begin{array}{c}2(0.9 \%) \\
-1.6\end{array}$ & $\begin{array}{c}31(13.7 \%) \\
-1.0\end{array}$ & & \\
\hline $4^{\circ}$ de ESO & $\begin{array}{c}353(81.0 \%) \\
2.6\end{array}$ & $\begin{array}{c}23(5.3 \%) \\
0.0\end{array}$ & $\begin{array}{c}12(2.8 \%) \\
0.7\end{array}$ & $\begin{array}{c}48(11.0 \%) \\
-3.3 \\
\end{array}$ & & \\
\hline $\begin{array}{l}1^{\circ} \text { de } \\
\text { Bachillerato }\end{array}$ & $\begin{array}{c}191(67.0 \%) \\
-4.1\end{array}$ & $\begin{array}{c}10(3.5 \%) \\
-1.5\end{array}$ & $\begin{array}{c}5(1.8 \%) \\
-0.7\end{array}$ & $\begin{array}{c}79(27.7 \%) \\
5.9\end{array}$ & & \\
\hline
\end{tabular}

Nota: $* *=p<.001 ; \mathrm{rc}=$ residuos corregidos. 


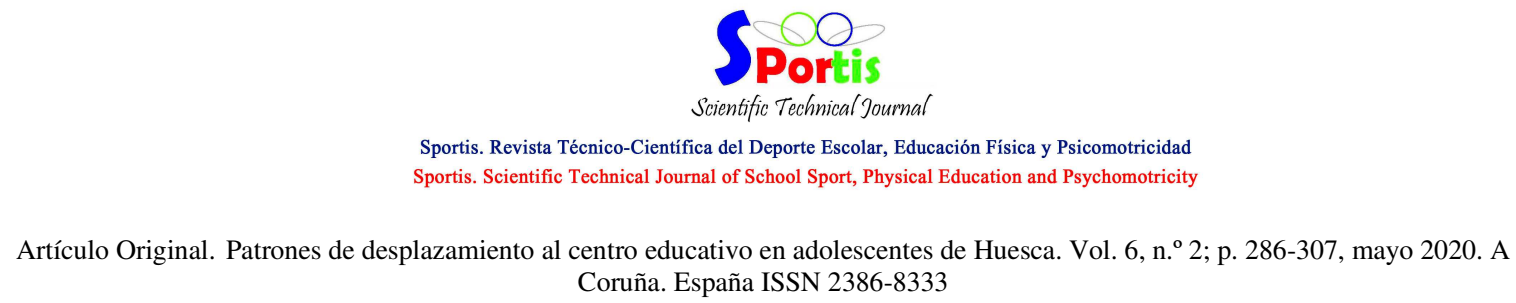

La Tabla 2 y 3 recogen los patrones de desplazamiento de ida y vuelta al centro escolar de manera independiente. Puede apreciarse que para ir al instituto un $78.5 \%$ de los estudiantes se desplaza andando $(n=1342)$, un $6 \%$ en bicicleta $(n=102)$, un $12.3 \%$ en coche $(n=211)$, un $0.6 \%$ en motocicleta $(n=10)$ y un $2.6 \%$ en autobús $(n=44)$, mientras que para volver del instituto, un $81.7 \%$ se desplaza andando $(\mathrm{n}=1397)$, un $6.2 \%$ en bicicleta $(\mathrm{n}=106)$, un $9.5 \%$ en coche $(n=163)$, un $0.6 \%$ en motocicleta $(n=10)$ y un $1.9 \%$ en autobús $(n=33)$ En relación con el género, se encontró tanto en el desplazamiento de ida como en de vuelta una asociación positiva entre andar al centro educativo y ser chica, así como entre ir en bicicleta y ser chico. En relación con el curso académico, desplazarse andando al centro educativo tanto en el desplazamiento de ida como en el de vuelta se asoció positivamente con los adolescentes de $4^{\circ}$ de ESO y negativamente con los de $1^{\circ}$ de Bachillerato. Por otro lado, desplazarse en bicicleta tanto en el desplazamiento de ida como en el de vuelta se asoció positivamente con los estudiantes de $1^{\circ}$ de Bachillerato y negativamente con los de $3^{\circ}$ de ESO. Asimismo, desplazarse en bicicleta se asoció negativamente con $4^{\circ}$ de ESO únicamente en el trayecto de ida. De igual modo, se encontró tanto en el desplazamiento de ida como en el de vuelta, una asociación positiva entre desplazarse en motocicleta y cursar $1^{\circ}$ de Bachillerato. Por último, cabe destacar que desplazarse en coche en la vuelta al centro educativo se asoció positivamente con los estudiantes de $1^{\circ}$ de Bachillerato y negativamente con los de $4^{\circ}$ de ESO (ver Tabla 2 y 3 ). 
Artículo Original. Patrones de desplazamiento al centro educativo en adolescentes de Huesca. Vol. 6, n. ${ }^{\text {2 }}$; p. 286-307, mayo 2020. A Coruña. España ISSN 2386-8333

Tabla 2. Patrones de desplazamiento en la ida al centro educativo: diferencias en función del género y el curso académico.

\begin{tabular}{|c|c|c|c|c|c|c|c|}
\hline & $\begin{array}{c}\text { Andando } \\
\mathrm{n}(\%) \\
\mathrm{rc}\end{array}$ & $\begin{array}{c}\text { Bicicleta } \\
\mathrm{n}(\%) \\
\mathrm{rc}\end{array}$ & $\begin{array}{c}\text { Coche } \\
\mathrm{n}(\%) \\
\text { rc }\end{array}$ & $\begin{array}{c}\text { Motocicleta } \\
\mathrm{n}(\%) \\
\mathrm{rc}\end{array}$ & $\begin{array}{c}\text { Autobús } \\
\text { n }(\%) \\
\text { rc }\end{array}$ & $x 2(g l)$ & $\begin{array}{c}\mathrm{V} \\
\text { Cramer }\end{array}$ \\
\hline Total & $1342(78.5 \%)$ & $102(6.0 \%)$ & $211(12.3 \%)$ & $10(0.6 \%)$ & $44(2.6 \%)$ & & \\
\hline \multicolumn{8}{|l|}{ Género } \\
\hline Chicos & $\begin{array}{c}607(74.6 \%) \\
-3.8\end{array}$ & $\begin{array}{c}82(10.1 \%) \\
6.7\end{array}$ & $\begin{array}{c}98(12.0 \%) \\
-0.2\end{array}$ & $\begin{array}{c}6(0.7 \%) \\
0.7\end{array}$ & $\begin{array}{c}21(2.6 \%) \\
-0.1\end{array}$ & \multirow[t]{2}{*}{$45.767(4)^{* *}$} & \multirow[t]{2}{*}{.165} \\
\hline Chicas & $\begin{array}{c}716(82.2 \%) \\
3.8 \\
\end{array}$ & $\begin{array}{c}20(2.3 \%) \\
-6.7 \\
\end{array}$ & $\begin{array}{c}108(12.4 \%) \\
0.2\end{array}$ & $\begin{array}{c}4(0.5 \%) \\
-0.7\end{array}$ & $\begin{array}{c}23(2.6 \%) \\
0.1\end{array}$ & & \\
\hline \multicolumn{8}{|l|}{ Curso académico } \\
\hline $1^{\circ}$ de ESO & $\begin{array}{c}237(78.0 \%) \\
-0.3 \\
\end{array}$ & $\begin{array}{c}23(7.6 \%) \\
1.3 \\
\end{array}$ & $\begin{array}{c}38(12.5 \%) \\
0.1\end{array}$ & $\begin{array}{c}1(0.3 \%) \\
-0.6 \\
\end{array}$ & $\begin{array}{c}5(1.6 \%) \\
-1.1 \\
\end{array}$ & \multirow{5}{*}{$56.898(16)^{* *}$} & \multirow{5}{*}{.091} \\
\hline $2^{\circ}$ de ESO & $\begin{array}{c}369(80.6 \%) \\
1.2 \\
\end{array}$ & $\begin{array}{c}20(4.4 \%) \\
-1.7 \\
\end{array}$ & $\begin{array}{c}53(11.6 \%) \\
-0.6 \\
\end{array}$ & $\begin{array}{c}2(0.4 \%) \\
-0.5 \\
\end{array}$ & $\begin{array}{c}14(3.1 \%) \\
0.8 \\
\end{array}$ & & \\
\hline $3^{\circ}$ de ESO & $\begin{array}{c}175(77.4 \%) \\
-0.4\end{array}$ & $\begin{array}{c}6(2.7 \%) \\
-2.3\end{array}$ & $\begin{array}{c}34(15.0 \%) \\
1.3\end{array}$ & $\begin{array}{c}2(0.9 \%) \\
0.6\end{array}$ & $\begin{array}{c}9(4.0 \%) \\
1.4\end{array}$ & & \\
\hline $4^{\circ}$ de ESO & $\begin{array}{c}365(83.7 \%) \\
3.1 \\
\end{array}$ & $\begin{array}{c}15(3.4 \%) \\
-2.6 \\
\end{array}$ & $\begin{array}{c}44(10.1 \%) \\
-1.7 \\
\end{array}$ & $\begin{array}{c}1(0.2 \%) \\
-1.1 \\
\end{array}$ & $\begin{array}{c}11(2.5 \%) \\
-0.1 \\
\end{array}$ & & \\
\hline $1^{\circ}$ de Bachillerato & $\begin{array}{c}196(68.8 \%) \\
-4.4\end{array}$ & $\begin{array}{c}38(13.3 \%) \\
5.7\end{array}$ & $\begin{array}{c}42(14.7 \%) \\
1.3\end{array}$ & $\begin{array}{c}4(1.4 \%) \\
2.0\end{array}$ & $\begin{array}{c}5(1.8 \%) \\
-1.0\end{array}$ & & \\
\hline
\end{tabular}

Nota: $* *=\mathrm{p}<.001 ; \mathrm{rc}=$ residuos corregidos.

Tabla 3. Patrones de desplazamiento en la vuelta al centro educativo: diferencias en función del género y el curso académico.

\begin{tabular}{|c|c|c|c|c|c|c|c|}
\hline & $\begin{array}{c}\text { Andando } \\
\mathrm{n}(\%) \\
\mathrm{rc}\end{array}$ & $\begin{array}{c}\text { Bicicleta } \\
\mathrm{n}(\%) \\
\mathrm{rc}\end{array}$ & $\begin{array}{c}\text { Coche } \\
\mathrm{n}(\%) \\
\text { rc }\end{array}$ & $\begin{array}{c}\text { Motocicleta } \\
\mathrm{n}(\%) \\
\text { rc }\end{array}$ & $\begin{array}{c}\text { Autobús } \\
\text { n }(\%) \\
\text { rc }\end{array}$ & $x 2(\mathrm{gl})$ & $\begin{array}{c}\mathrm{V} \\
\text { Cramer }\end{array}$ \\
\hline Total & $1397(81.7 \%)$ & $106(6.2 \%)$ & $163(9.5 \%)$ & $10(0.6 \%)$ & $33(1.9 \%)$ & & \\
\hline \multicolumn{8}{|l|}{ Género } \\
\hline Chicos & $\begin{array}{c}631(77.5 \%) \\
-4.1 \\
\end{array}$ & $\begin{array}{c}84(10.3 \%) \\
6.6 \\
\end{array}$ & $\begin{array}{c}76(9.3 \%) \\
-0.5 \\
\end{array}$ & $\begin{array}{c}6(0.7 \%) \\
0.7 \\
\end{array}$ & $\begin{array}{c}17(2.1 \%) \\
0.4\end{array}$ & \multirow[t]{2}{*}{$44.533(4) * *$} & \multirow[t]{2}{*}{.163} \\
\hline Chicas & $\begin{array}{c}742(85.2 \%) \\
4.1 \\
\end{array}$ & $\begin{array}{c}22(2.5 \%) \\
-6.6 \\
\end{array}$ & $\begin{array}{c}87(10.0 \%) \\
0.5 \\
\end{array}$ & $\begin{array}{c}4(0.5 \%) \\
-0.7 \\
\end{array}$ & $\begin{array}{c}16(1.8 \%) \\
-0.4 \\
\end{array}$ & & \\
\hline \multicolumn{8}{|l|}{ Curso académico } \\
\hline $1^{\circ}$ de ESO & $\begin{array}{c}255(83.9 \%) \\
1.1 \\
\end{array}$ & $\begin{array}{c}22(7.2 \%) \\
0.8 \\
\end{array}$ & $\begin{array}{c}23(7.6 \%) \\
-1.3 \\
\end{array}$ & $\begin{array}{c}2(0.7 \%) \\
0.2 \\
\end{array}$ & $\begin{array}{c}2(0.7 \%) \\
-1.8 \\
\end{array}$ & \multirow{5}{*}{$68.479(16) * *$} & \multirow{5}{*}{.100} \\
\hline $2^{\circ}$ de ESO & $\begin{array}{c}372(81.2 \%) \\
-0.3\end{array}$ & $\begin{array}{c}20(4.4 \%) \\
-1.9 \\
\end{array}$ & $\begin{array}{c}53(11.6 \%) \\
1.7 \\
\end{array}$ & $\begin{array}{c}0(0.0 \%) \\
-1.9\end{array}$ & $\begin{array}{c}13(2.8 \%) \\
1.6\end{array}$ & & \\
\hline $3^{\circ}$ de ESO & $\begin{array}{c}193(85.4 \%) \\
1.5 \\
\end{array}$ & $\begin{array}{c}5(2.2 \%) \\
-2.7 \\
\end{array}$ & $\begin{array}{c}21(9.3 \%) \\
-0.1 \\
\end{array}$ & $\begin{array}{c}2(0.9 \%) \\
0.6 \\
\end{array}$ & $\begin{array}{c}5(2.2 \%) \\
0.3 \\
\end{array}$ & & \\
\hline $4^{\circ}$ de ESO & $\begin{array}{c}376(86.2 \%) \\
2.8\end{array}$ & $\begin{array}{c}19(4.4 \%) \\
-1.9\end{array}$ & $\begin{array}{c}29(6.7 \%) \\
-2.4 \\
\end{array}$ & $\begin{array}{c}2(0.5 \%) \\
-0.4\end{array}$ & $\begin{array}{c}10(2.3 \%) \\
0.6\end{array}$ & & \\
\hline $1^{\circ}$ de Bachillerato & $\begin{array}{c}201(70.5 \%) \\
-5.4\end{array}$ & $\begin{array}{c}40(14.0 \%) \\
6.0\end{array}$ & $\begin{array}{c}37(13.0 \%) \\
2.2 \\
\end{array}$ & $\begin{array}{c}4(1.4 \%) \\
2.0 \\
\end{array}$ & $\begin{array}{c}3(1.1 \%) \\
-1.2 \\
\end{array}$ & & \\
\hline
\end{tabular}

Nota: $* *=p<.001 ; \mathrm{rc}=$ residuos corregidos. 


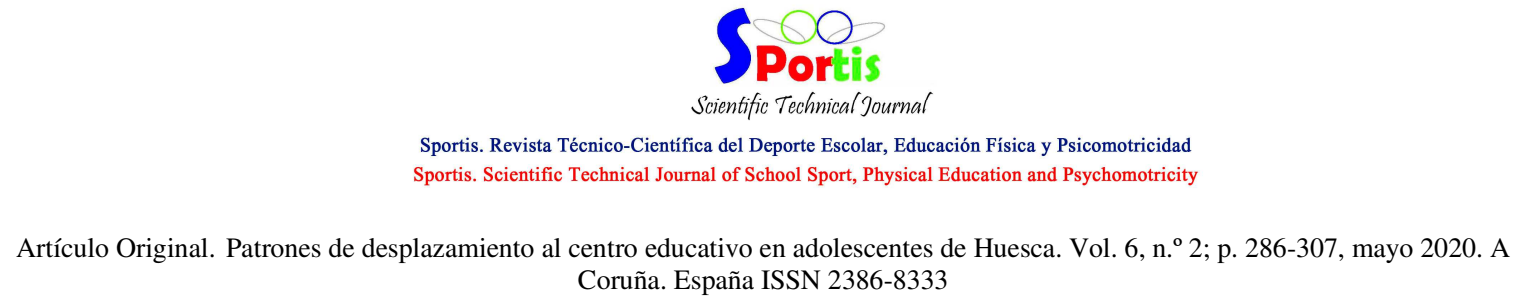
Coruña. España ISSN 2386-8333

\section{Discusión}

El primer objetivo de este estudio fue describir el tipo de desplazamiento utilizado para ir y volver al centro educativo en adolescentes de la ciudad de Huesca. Los resultados obtenidos señalaron que tres de cada cuatro adolescentes se desplazaron activamente (i.e., andando o en bicicleta) tanto en la ida como en la vuelta, mientras que uno de cada cuatro se desplazó pasivamente (i.e., vehículo motorizado) en ambos trayectos. El porcentaje de adolescentes que se desplazó activamente en el presente estudio es superior al encontrado en la mayoría de estudios a nivel internacional y nacional (Denstel et al., 2015; Masoumi et al., 2017; Yang, Ivey, Levy, Royne, y Klesges, 2016). Por ejemplo, un estudio llevado a cabo en más de 100.000 adolescentes asiáticos señaló que el 42.1\% se desplazó activamente (Uddin et al., 2019). En España, los patrones de desplazamiento activo en adolescentes oscilan desde un $46 \%$, en un estudio realizado en todas las comunidades autónomas, y las ciudades de Ceuta y Melilla (Sanz-Arazuri et al., 2017) hasta un 71\% (Aparicio-Ugarriza et al., 2020) en un estudio llevado a cabo en una muestra representativa de niños y adolescentes españoles. El alto porcentaje de adolescentes que se desplazan activamente en el presente estudio podría deberse a las pequeñas dimensiones de la ciudad de Huesca, lo que facilita que las distancias entre los hogares y los centros educativos sean cortas. Revisiones sistemáticas previas han señalado que la distancia al centro escolar es uno de los factores que más se ha relacionado con el desplazamiento activo (Ikeda et al., 2018; Rojas y Wong, 2017; Rothman, Macpherson, Ross, y Buliung, 2018). En esta línea, un estudio realizado en España señaló que la máxima distancia que los adolescentes están dispuestos a andar al centro educativo es de 1.350 metros (Rodríguez-López et al., 2016), coincidiendo con el umbral de distancia identificado también para la ciudad de Huesca, lo cual podría facilitar que los jóvenes se desplacen activamente en dicha ciudad (Zaragoza, Corral, Estrada, Abós, y Aibar, 2019). Dado que una reciente revisión sistemática identificó la seguridad del tráfico como uno de las principales barreras percibidas por los padres en el desplazamiento de sus hijos (Aranda-Balboa, HuertasDelgado, Herrador-Colmenero, Cardon, y Chillón, 2019), el actual plan de movilidad urbana sostenible en Huesca, que incluye 32 hectáreas de zonas peatonales (i.e., 100 calles y plazas) y $23.78 \mathrm{~m}^{2}$ de zonas verdes por habitante, podría haber favorecido una mayor percepción de 


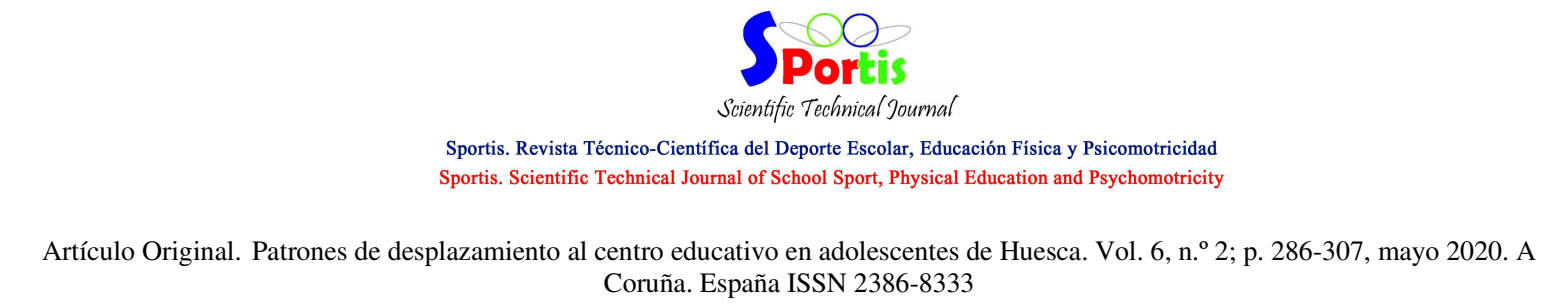

seguridad en las familias y, por tanto, una mayor movilidad activa de los adolescentes (Ayuntamiento de Huesca, 2018). No obstante, a pesar de que un elevado porcentaje de los adolescentes del presente estudio se desplaza activamente en ambos trayectos, consideramos necesario seguir reforzando la promoción de programas escolares que incentiven el desplazamiento activo dado el pequeño tamaño y las características favorables de movilidad que tiene la ciudad de Huesca.

Concretamente, la mayoría de los adolescentes manifestó desplazarse andando para ir y volver del instituto, seguido del coche, la bicicleta, el autobús y, por último, la motocicleta. El porcentaje de adolescentes que se desplazó caminando es muy superior al encontrado en la literatura internacional y nacional (Masoumi et al., 2017). Para conocimiento de los autores, el porcentaje de jóvenes españoles que caminan al centro educativo oscila desde un $44 \%$ (Sanz-Arazuri et al., 2017) hasta un 71\% (Aparicio-Ugarriza et al., 2020). Sin embargo, el porcentaje de adolescentes que se desplazó en bicicleta en el presente estudio es inferior al encontrado en la mayoría de los estudios realizados en algunos países europeos. Por ejemplo, en el estudio ENERGY, realizado en 8 países europeos, se encontró que el $21.4 \%$ de los niños entre 10 y 12 años se desplazaba en bicicleta al centro educativo (Te Velde et al., 2017). No obstante, cabe destacar que, en la mayoría de los estudios realizados en otras ciudades españolas, se ha encontrado un porcentaje más bajo de adolescentes que se desplaza en bicicleta (Chillón et al., 2013; Herrador-Colmenero et al., 2019; Martínez-Gómez et al., 2011; Te Velde et al., 2017). En este sentido, el pequeño tamaño de la ciudad de Huesca, la orografía favorable y la extensa red de carriles bici podrían explicar que los adolescentes oscenses se desplacen más en bicicleta que en otras ciudades españolas. En esta línea, una revisión sistemática previa también mostró que en las zonas en la que los barrios son peatonales, existen parques y tienen más carriles de bici, los jóvenes adoptan patrones de desplazamiento más activos al centro educativo (Smith et al., 2017). Futuras investigaciones deberían analizar las barreras percibidas por los adolescentes para desplazarse en bicicleta al centro educativo en Huesca, dada las características favorables de la ciudad. De igual modo, parece necesario que futuros estudios en la ciudad de Huesca examinen la distancia objetiva (e.g., Google Earth) entre el domicilio y el instituto ya que las pequeñas distancias entre ambos lugares podrían explicar un menor uso de la bicicleta al centro educativo (e.g., si un 


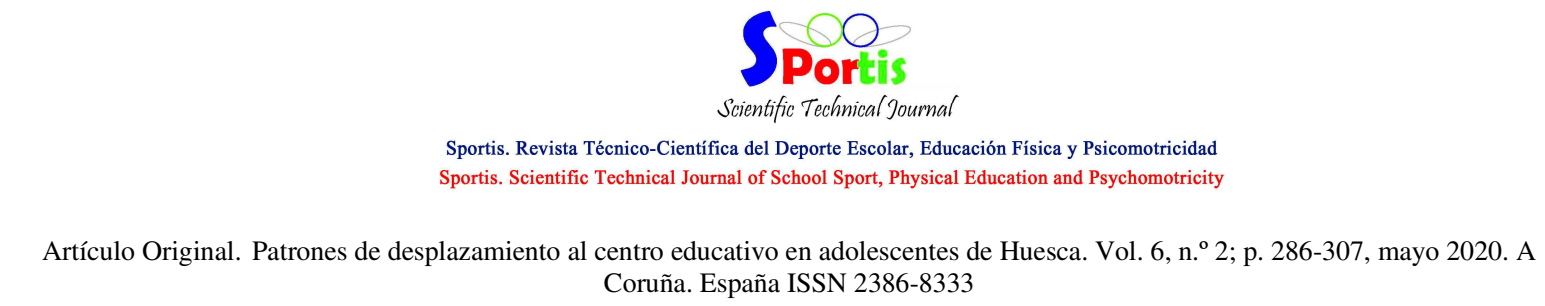

adolescente vive a menos de 5 minutos del centro educativo es posible que decida no usar la bicicleta ya que es más cómodo desplazarse caminando). Por último, cabe destacar que el porcentaje de adolescentes oscenses que se desplazan en autobús al centro educativo es muy inferior respecto a otros estudios realizados en jóvenes españoles (Herrador-Colmenero et al., 2019). Este hecho podría deberse a que la ciudad de Huesca solo dispone de tres líneas de autobús con una frecuencia de paso muy baja. En base a los resultados encontrados, resulta necesario diseñar e implementar programas escolares y extraescolares que promuevan el uso habitual de la bicicleta dado que este modo de desplazamiento produce un mayor incremento de los niveles de AFMV y de la condición física en comparación con caminar (Larouche et al., 2014).

En relación con el modo de desplazamiento en la ida y vuelta, cabe destacar que, en sintonía con el limitado número de estudios nacionales e internacionales existente (HerradorColmenero et al., 2014; Herrador-Colmenero et al., 2019; Larsen et al., 2012; Ruiz-Ariza et al., 2015), un 3\% más de los adolescentes oscenses manifestó desplazarse andando al centro educativo en el trayecto de vuelta en comparación con el de ida, mientras que el desplazamiento en coche fue un $3 \%$ mayor en la ida que en la vuelta. Esto podría deberse a que algunos padres llevan a sus hijos al centro educativo en el trayecto de ida cuando van al trabajo (Ahern et al., 2017). Otra posible explicación podría ser debido a la falta de tiempo por las mañanas provocada por una menor duración de sueño de los jóvenes a causa del elevado tiempo de pantalla, especialmente antes de acostarse (Mei et al., 2018). En esta línea, un estudio señaló que aquellos adolescentes que cumplen las recomendaciones de duración de sueño se desplazan más activamente al centro educativo (Villa-González, Huertas-Delgado, Chillón, Ramírez-Vélez, y Barranco-Ruiz, 2019). Otra razón para usar más el coche en el trayecto de ida podría deberse a que los jóvenes tienen que llegar al colegio con puntualidad, mientras que en la vuelta no tienen tan prisa y podrían preferir volver caminando junto a sus compañeros (Yang et al., 2016). Futuras investigaciones deberían seguir profundizando en las razones por las que los jóvenes utilizan diferentes modos de desplazamiento en la ida y en la vuelta al centro escolar.

El segundo objetivo de este trabajo fue analizar la asociación entre el tipo de desplazamiento utilizado para ir y volver al centro educativo y el género y el curso académico

Para citar este artículo utilice la siguiente referencia: Simón-Montañés, L.; Aibar, A.; Abós, A.; García-González, L.; Sevil-Serrano, J. (2020).

Patrones de desplazamiento al centro educativo en adolescentes de Huesca. Sportis Sci J, 6 (2), 286-307. DOI: https://doi.org/10.17979/sportis.2020.6.2.5975 


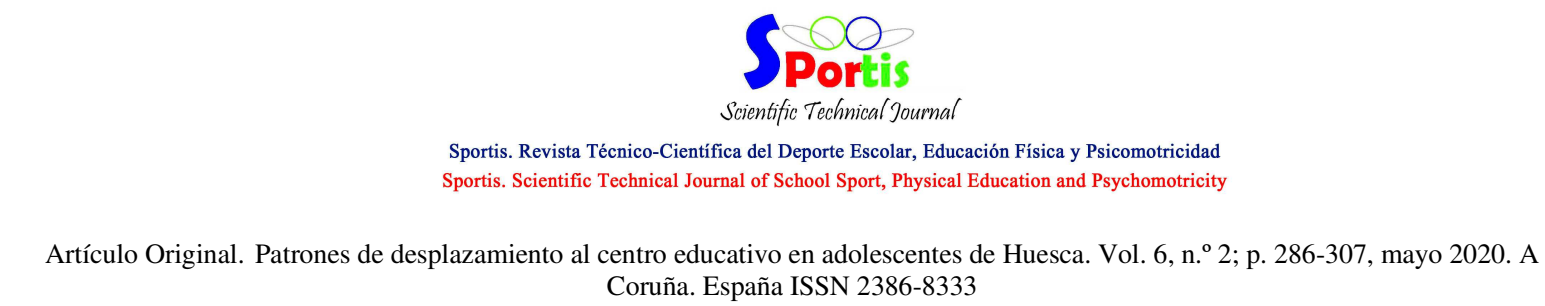

de los jóvenes. En relación con el género, se encontró tanto en el desplazamiento de ida como en el de vuelta una asociación positiva entre andar al centro educativo y ser chica, así como entre ir en bicicleta y ser chico. Estos resultados están en línea de una revisión sistemática previa que señaló que los chicos se desplazaban más en bicicleta, mientras que las chicas caminando (Rojas y Wong, 2017). Sin embargo, estos resultados no están en sintonía con los hallados en la mayor parte de estudios llevados a cabo en diferentes ciudades españolas donde el porcentaje de chicas y chicos que se desplaza caminando y en bicicleta es prácticamente el mismo (Chillón et al., 2013; Martínez-Gómez et al., 2011; Ruiz-Ariza et al., 2015; SanzArazuri, et al., 2017). Estudios previos han analizado algunas de las posibles causas que podrían explicar el menor porcentaje de chicas que se desplaza en bicicleta a la escuela. Por ejemplo, un estudio cualitativo llevado a cabo por Frater y Kingham (2018) en chicas adolescentes señaló que no suelen desplazarse en bicicleta porque lo ven como un deporte masculino y ellas quieren sentirse femeninas y socializarse. Asimismo, señalan la falta de apoyo (e.g., no ir en bicicleta porque es peligroso) o normas impuestas (e.g., ponerse el casco o los reflectantes) por los padres y amigos como barreras para desplazarse en bicicleta. Por último, la falta de competencia y habilidad en el desplazamiento en bicicleta, así como las preocupaciones por su seguridad personal, son otras dos de las razones por las que manifiestan que no usan la bicicleta. Por lo tanto, parece necesario promover desde el ámbito educativo el desplazamiento en bicicleta involucrando a todos los agentes de la comunidad educativa (e.g., profesorado, familia, alumnado, etc.). El diseño de estrategias específicas que permitan disminuir o eliminar las barreras percibidas por los adolescentes, especialmente por las chicas, se abre como una importante línea de investigación.

En relación con el curso académico, cabe destacar que tanto en el desplazamiento de ida como en el de vuelta desplazarse andando al centro educativo se asoció positivamente con los adolescentes de $4^{\circ}$ de ESO y negativamente con $1^{\circ}$ de Bachillerato. Por otro lado, desplazarse en bicicleta se asoció positivamente con $1^{\circ}$ de Bachillerato y negativamente con $3^{\circ}$ de ESO tanto en el trayecto de ida y vuelta. Para conocimiento de los autores, no existe en la literatura nacional e internacional estudios similares que analicen el modo de desplazamiento en función del curso académico. Sin embargo, algunas investigaciones han descrito los patrones de desplazamiento en función de la edad o grupos de edad durante la

Para citar este artículo utilice la siguiente referencia: Simón-Montañés, L.; Aibar, A.; Abós, A.; García-González, L.; Sevil-Serrano, J. (2020).

Patrones de desplazamiento al centro educativo en adolescentes de Huesca. Sportis Sci J, 6 (2), 286-307. DOI: https://doi.org/10.17979/sportis.2020.6.2.5975 


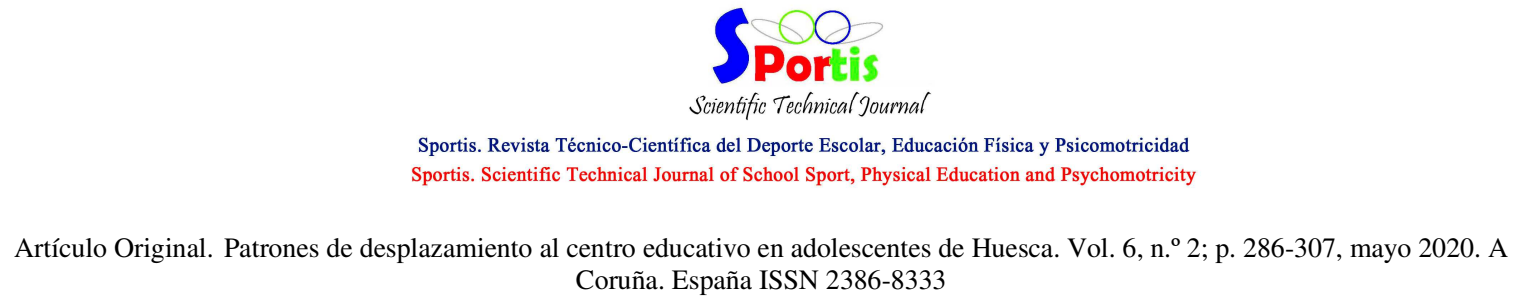

adolescencia. A nivel internacional no existen unos resultados concluyentes en la literatura científica (Rothman et al., 2018), encontrándose estudios en los que desplazarse caminando y en bicicleta al instituto fue mayor en adolescentes jóvenes que en adolescentes más mayores (Kontou et al., 2019; Uddin et al., 2019) y viceversa (Uddin et al., 2019). Para conocimiento de los autores, solo existe un estudio a nivel nacional en el que se examinen los patrones de desplazamiento activo en función de la edad. Dicho estudio señaló que los jóvenes de la primera etapa de la adolescencia (i.e., 13 a 15 años) se desplazaban más activamente que los jóvenes de 17 a 18 años. Sin embargo, a diferencia del presente estudio, en dicha investigación no se analizó separadamente el tipo de desplazamiento activo (i.e., caminar e ir en bicicleta) (Chillón et al., 2009). Aunque los resultados de la literatura no son concluyentes, la asociación positiva entre usar la bicicleta y cursar $1^{\circ}$ de Bachillerato podría estar justificada por una mayor madurez, una mayor habilidad, percepción de competencia y seguridad con la bicicleta, un mayor conocimiento de las normas de circulación vial y las señales, así como una menor percepción de barreras por parte de los padres (Aranda-Balboa et al., 2019; Mandic et al., 2018; Rojas y Wong, 2017). Por lo tanto, parece necesario el diseño de estrategias específicas para promover el desplazamiento activo en bicicleta en el alumnado de ESO. Asimismo, es importante seguir investigando en las razones por las que los jóvenes deciden desplazarse de uno u otro modo en función del curso académico.

Una de las fortalezas del presente estudio es que participaron aproximadamente 1.709 estudiantes, incluyendo seis de los ocho institutos públicos y concertados de la ciudad de Huesca. Asimismo, participaron la totalidad de los cursos que comprenden la etapa educativa de ESO y $1^{\circ}$ Bachillerato, cursos donde la asignatura de Educación Física es obligatoria según el currículo. Para conocimiento de los autores, estudios previos no han analizado los patrones de desplazamiento activo de los adolescentes en función del curso académico, siendo esta la principal aportación de esta investigación. Del mismo modo, dado que la mayoría de estudios previos no han examinado el modo de desplazamiento de ida y vuelta al centro educativo (Herrador-Colmenero et al., 2014), este análisis de ambos trayectos supone otra importante contribución a la literatura.

Sin embargo, esta investigación presenta una serie de limitaciones que es necesario reconocer. En base a ellas, se plantean una serie de prospectivas de estudio para seguir

Para citar este artículo utilice la siguiente referencia: Simón-Montañés, L.; Aibar, A.; Abós, A.; García-González, L.; Sevil-Serrano, J. (2020). Patrones de desplazamiento al centro educativo en adolescentes de Huesca. Sportis Sci J, 6 (2), 286-307. DOI: https://doi.org/10.17979/sportis.2020.6.2.5975 


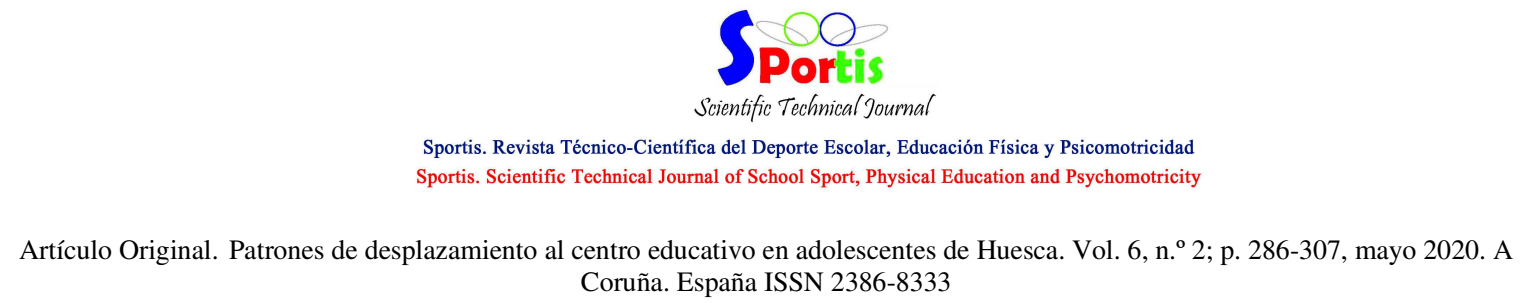

avanzando en esta línea de investigación. En primer lugar, este trabajo se corresponde con un estudio transversal. Por ello, son necesarios estudios longitudinales o por cohortes que nos permitan identificar cómo evolucionan los patrones de desplazamiento de los adolescentes a lo largo del tiempo. En segundo lugar, el cuestionario utilizado contempló únicamente el modo de desplazamiento habitual para ir y volver del centro educativo. Por lo tanto, no se tuvo en cuenta la posibilidad de que este variase cada uno de los días lectivos de la semana (e.g., que el lunes y martes se desplazasen en bicicleta y el miércoles, jueves o viernes caminando) o que los adolescentes combinasen dos posibles modos de desplazamiento al centro educativo (e.g., que fueran andando hasta la parada de autobús y luego utilizaran este medio de transporte para llegar al instituto). Futuros estudios deberían evaluar no solo la ida y vuelta al instituto sino también el modo de desplazamiento diario en una semana de duración. De igual modo, sería conveniente permitir la posibilidad de cumplimentar varios modos de desplazamiento diario para tener una medida más real de la forma de desplazamiento de cada joven. En tercer lugar, se utilizó una muestra por conveniencia por lo que, aunque la muestra es muy alta, los resultados encontrados no son representativos de los adolescentes de la ciudad de Huesca. Por ello, futuros estudios deberían evaluar los patrones de desplazamiento en una muestra representativa de adolescentes de esta localidad. Por último, dado que solo se evaluó el modo de desplazamiento a través de un cuestionario autoadministrado, sería conveniente complementar este tipo de estudios con una medida más objetiva como acelerómetros y GPS para conocer los minutos de AFMV acumulados en el recorrido y la distancia existente entre el hogar y el centro educativo. Asimismo, futuros estudios podrían complementar los datos obtenidos con metodología cualitativa (e.g., grupos de discusión) para justificar algunos de los resultados hallados.

\section{Conclusiones}

La mayoría de los adolescentes de ESO y $1^{\circ}$ de Bachillerato de la ciudad de Huesca se desplazan de forma activa para ir y volver del instituto. Aunque tres de cada cuatro adolescentes se desplazan caminando al centro escolar, la mayoría no utiliza la bicicleta como medio de desplazamiento para ir y volver al centro educativo. Asimismo, se encontraron asociaciones positivas entre estudiar $1^{\circ}$ de Bachillerato y utilizar la bicicleta y estudiar $4^{\circ}$ de

Para citar este artículo utilice la siguiente referencia: Simón-Montañés, L.; Aibar, A.; Abós, A.; García-González, L.; Sevil-Serrano, J. (2020).

Patrones de desplazamiento al centro educativo en adolescentes de Huesca. Sportis Sci J, 6 (2), 286-307. DOI: https://doi.org/10.17979/sportis.2020.6.2.5975 


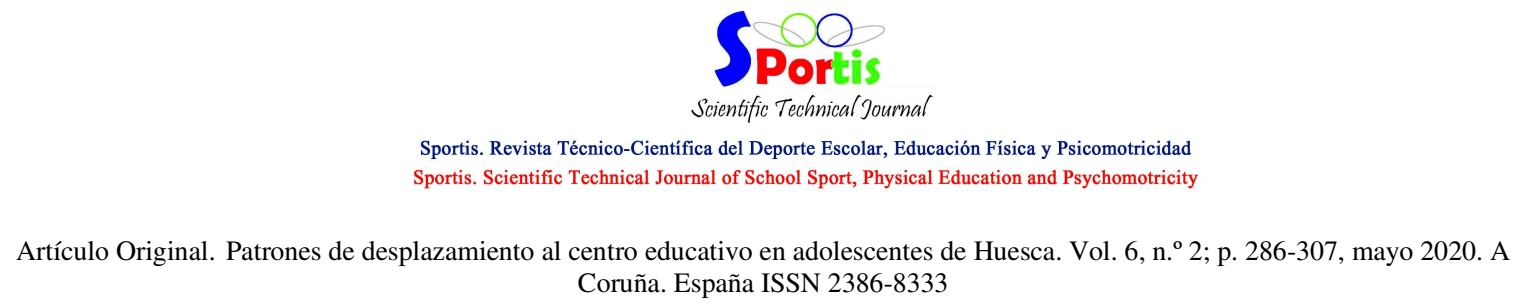

ESO y desplazarse caminando. Dadas las dimensiones y las características favorables de movilidad en la ciudad de Huesca, parecen necesarios programas escolares que promuevan el desplazamiento andando al centro educativo, especialmente en $1^{\circ}$ de Bachillerato. Del mismo modo, resulta necesaria la promoción de la bicicleta como modo de desplazamiento al centro educativo debido a su bajo uso entre los adolescentes, especialmente en las chicas y en los estudiantes de ESO.

\section{Referencias bibliográficas}

Abarca, A., Gallardo, L., Generelo, E., Julián, J.A., y Zaragoza, J. (2019). ¿Difieren las influencias personales, sociales,ambientales y psicológicas en la actividad física en base al tipo desplazamiento al centro escolar? Universitas Psychologica, 18 (4).

Ahern, S. M., Arnott, B., Chatterton, T., de Nazelle, A., Kellar, I., y McEachan, R. R. (2017). Understanding parents' school travel choices: a qualitative study using the Theoretical Domains Framework. Journal of Transport \& Health, 4, 278-293. DOI: https://doi.org/10.1016/j.jth.2016.11.001

Aparicio-Ugarriza, R., Mielgo-Ayuso, J., Ruiz, E., Ávila, J. M., Aranceta-Bartrina, J., Gil, Á., ... y González-Gross, M. (2020). Active Commuting, Physical Activity, and Sedentary Behaviors in Children and Adolescents from Spain: Findings from the ANIBES Study. International Journal of Environmental Research and Public Health, 17(2). DOI: https://doi.org/10.3390/ijerph17020668

Aranda-Balboa, M. J., Huertas-Delgado, F. J., Herrador-Colmenero, M., Cardon, G., y Chillón, P. (2019). Parental barriers to active transport to school: a systematic review. International Journal of Public Health, 1-12. DOI: https://doi.org/10.1007/s00038019-01313-1

Ayuntamiento de Huesca. (2018). La ciudad, zona peatonal. Recuperado de: https://www.huesca.es/la-ciudad/trafico-y-movilidad/zona-peatonal

Booth, V., Rowlands, A., y Dollman, J. (2019). Physical activity trends in separate contexts among south australian older children (10-12 y) and early adolescents (13-15 y) from

Para citar este artículo utilice la siguiente referencia: Simón-Montañés, L.; Aibar, A.; Abós, A.; García-González, L.; Sevil-Serrano, J. (2020). Patrones de desplazamiento al centro educativo en adolescentes de Huesca. Sportis Sci J, 6 (2), 286-307. DOI: https://doi.org/10.17979/sportis.2020.6.2.5975 


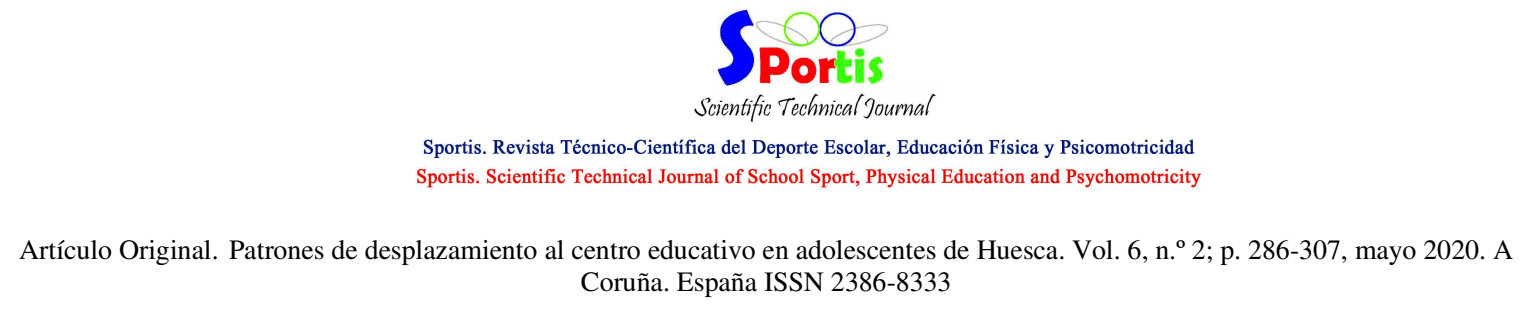
Coruña. España ISSN 2386-8333

1985 to 2013. Pediatric Exercise Science, 31(3), 341-347. DOI: https://doi.org/10.1123/pes.2018-0082

Chillón, P., Herrador-Colmenero, M., Migueles, J. H., Cabanas-Sánchez, V., FernándezSantos, J. R., Veiga, Ó. L., ... Castro-Piñero, J. (2017). Convergent validation of a questionnaire to assess the mode and frequency of commuting to and from school. Scandinavian Journal of Public Health, 45(6), 612-620. DOI: https://doi.org/10.1177/1403494817718905

Chillón, P., Martínez-Gómez, D., Ortega, F. B., Pérez-López, I. J., Díaz, L. E., Veses, A. M., ... Delgado-Fernández, M. (2013). Six-year trend in active commuting to school in Spanish adolescents. International Journal of Behavioral Medicine, 20(4), 529-537. DOI: https://doi.org/10.1007/s12529-012-9267-9

Chillón, P., Ortega, F. B., Ruiz, J. R., Pérez, I. J., Martín-Matillas, M., Valtueña, J., ... Tercedor, P. (2009). Socio-economic factors and active commuting to school in urban Spanish adolescents: the AVENA study. The European Journal of Public Health, 19(5), 470-476. DOI: https://doi.org/10.1093/eurpub/ckp048

Cohen, J. (1988). Statistical power analysis for the behavioral sciences. Hillsdale, NJ: Lawrence Erlbaum Associates. DOI: https://doi.org/10.4324/9780203771587

Denstel, K. D., Broyles, S. T., Larouche, R., Sarmiento, O. L., Barreira, T. V., Chaput, J. P., ... Kurpad, A. (2015). Active school transport and weekday physical activity in 9-11year-old children from 12 countries. International Journal of Obesity Supplements, 5(2), 100-106. DOI: https://doi.org/doi:10.1038/ijosup.2015.26

Frater, J., y Kingham, S. (2018). Gender equity in health and the influence of intrapersonal factors on adolescent girls' decisions to bicycle to school. Journal of Transport Geography, 71, 130-138. DOI: https://doi.org/10.1016/j.jtrangeo.2018.07.011

Gössling, S., Choi, A., Dekker, K., y Metzler, D. (2019). The social cost of automobility, cycling and walking in the European Union. Ecological Economics, 158, 65-74. DOI: https://doi.org/10.1016/j.ecolecon.2018.12.016

Guthold, R., Stevens, G. A., Riley, L. M., y Bull, F. C. (2020). Global trends in insufficient physical activity among adolescents: a pooled analysis of 298 population-based

Para citar este artículo utilice la siguiente referencia: Simón-Montañés, L.; Aibar, A.; Abós, A.; García-González, L.; Sevil-Serrano, J. (2020). Patrones de desplazamiento al centro educativo en adolescentes de Huesca. Sportis Sci J, 6 (2), 286-307. DOI: https://doi.org/10.17979/sportis.2020.6.2.5975 


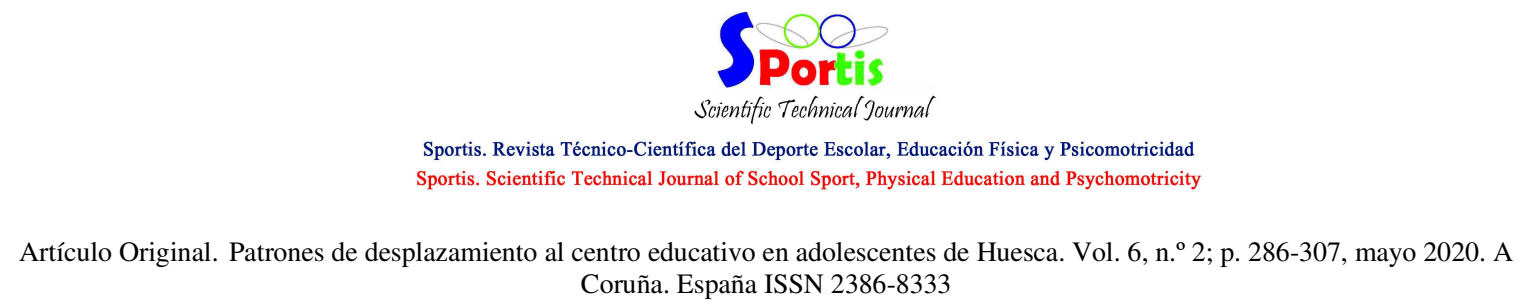

surveys with 1. 6 million participants. The Lancet Child \& Adolescent Health, 4(1), 23-35. DOI: https://doi.org/10.1016/S2352-4642(19)30323-2

Herrador-Colmenero, M., Escabias, M., Ortega, F. B., McDonald, N. C., y Chillón, P. (2019). Mode of Commuting TO and FROM School: a similar or different pattern? Sustainability, 11(4), 1026. DOI: https://doi.org/10.3390/su11041026

Herrador-Colmenero, M., Perez-Garcia, M., Ruiz, J. R., y Chillon, P. (2014). Assessing modes and frequency of commuting to school in youngsters: a systematic review. Pediatric Exercise Sciences, 26(3), 291-341. DOI: https://doi.org/10.1123/pes.20130120

Ikeda, E., Stewart, T., Garrett, N., Egli, V., Mandic, S., Hosking, J., ... Moore, A. (2018). Built environment associates of active school travel in New Zealand children and youth: A systematic meta-analysis using individual participant data. Journal of Transport \& Health, 9, 117-131. DOI: https://doi.org/10.1016/j.jth.2018.04.007

Kemp, B. J., Cliff, D. P., Chong, K. H., y Parrish, A. M. (2019). Longitudinal changes in domains of physical activity during childhood and adolescence: A systematic review. Journal of Science and Medicine in Sport, 22(6), 695-701. DOI: https://doi.org/10.1016/j.jsams.2018.12.012

Kontou, E., McDonald, N. C., Brookshire, K., Pullen-Seufert, N. C., y LaJeunesse, S. (2019). US active school travel in 2017: prevalence and correlates. Preventive Medicine Reports, 17, 101024. DOI: https://doi.org/10.1016/j.pmedr.2019.101024

Larouche, R., Saunders, T. J., John Faulkner, G. E., Colley, R., y Tremblay, M. (2014). Associations between active school transport and physical activity, body composition, and cardiovascular fitness: a systematic review of 68 studies. Journal of Physical Activity and Health, 11(1), 206-227. DOI: https://doi.org/10.1123/jpah.2011-0345

Larsen, K., Gilliland, J., y Hess, P. M. (2012). Route-based analysis to capture the environmental influences on a child's mode of travel between home and school. Annals of the Association of American Geographers, 102(6), 1348-1365. DOI: https://doi.org/10.1080/00045608.2011.627059

Mandic, S., Flaherty, C., Pocock, T., Kek, C. C., McArthur, S., Ergler, C., ... Bengoechea, E. G. (2018). Effects of cycle skills training on children's cycling-related knowledge, 


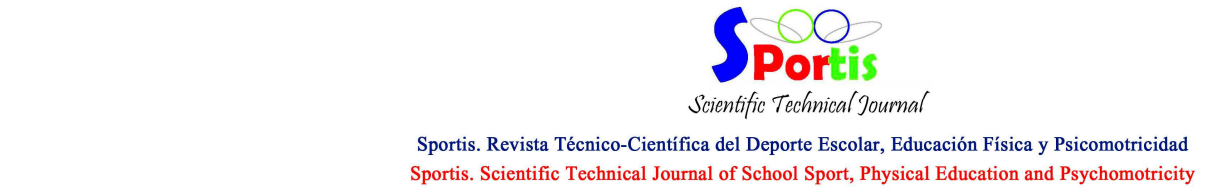

Artículo Original. Patrones de desplazamiento al centro educativo en adolescentes de Huesca. Vol. 6, n. ${ }^{\text {2 }}$; p. 286-307, mayo 2020. A Coruña. España ISSN 2386-8333

confidence and behaviours. Journal of Transport \& Health, 8, 271-282. DOI: https://doi.org/10.1016/j.jth.2017.12.010

Martínez-Gómez, D., Veiga, O. L., Gómez-Martínez, S., Zapatera, B., Calle, M. E., y Marcos, A. (2011). Behavioural correlates of active commuting to school in Spanish adolescents: the AFINOS (physical activity as a preventive measure against overweight, obesity, infections, allergies, and cardiovascular disease risk factors in adolescents) study. Public Health Nutrition, 14(10), 1779-1786. DOI: https://doi.org/10.1017/S1368980010003253

Masoumi, H. E., Zanoli, G., Papageorgiou, A., Smaga, S., Miloš, A., van Rooijen, M., ... Çağan, B. (2017). Patterns of children's travel to school, their body weight, spatial factors, and perceptions: a survey on nine European cities. GeoScape, 11(2), 52-75. DOI: https://doi.org/10.1515/geosc-2017-0005

Mei, X., Zhou, Q., Li, X., Jing, P., Wang, X., \& Hu, Z. (2018). Sleep problems in excessive technology use among adolescent: a systemic review and meta-analysis. Sleep Science and Practice, 2(1), 9. DOI: https://doi.org/10.1186/s41606-018-0028-9

Organización de las Naciones Unidas, Asamblea. General. (2015). Transformar nuestro mundo: la Agenda 2030 para el Desarrollo Sostenible. Recuperado de: https://unctad.org/meetings/es/SessionalDocuments/ares70d1_es.pdf

Poitras, V. J., Gray, C. E., Borghese, M. M., Carson, V., Chaput, J. P., Janssen, I., ... Tremblay, M. S. (2016). Systematic review of the relationships between objectively measured physical activity and health indicators in school-aged children and youth. Applied Physiology, Nutrition, and Metabolism, 41(6), 197-239. DOI: https://doi.org/10.1139/apnm-2015-0663.

Red de ciudades por la bicicleta (2019). La bicicleta ayuda a conseguir 12 de los 17 Objetivos de Desarrollo Sostenible (ODS) de la ONU. Recuperado de: https://www.ciudadesporlabicicleta.org/2019/10/18/la-bicicleta-ayuda-a-conseguir-12de-los-17-objetivos-de-desarrollo-sostenible-ods-de-la-onu/

Rodríguez-López, C., Vila-González, E., Herrador-Colmenero, M., Ortega, F. B., y Chillón, P. (2016). Desplazamiento activo al colegio: ¿qué distancia están dispuestos a andar nuestros jóvenes? Habilidad Motriz: Revista de Ciencias de la Actividad Física y del

Para citar este artículo utilice la siguiente referencia: Simón-Montañés, L.; Aibar, A.; Abós, A.; García-González, L.; Sevil-Serrano, J. (2020).

Patrones de desplazamiento al centro educativo en adolescentes de Huesca. Sportis Sci J, 6 (2), 286-307. DOI: https://doi.org/10.17979/sportis.2020.6.2.5975 


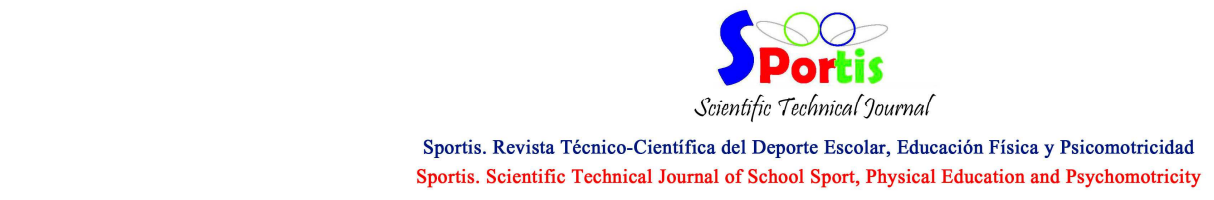

Artículo Original. Patrones de desplazamiento al centro educativo en adolescentes de Huesca. Vol. 6, n. ${ }^{\text {2 }}$; p. 286-307, mayo 2020. A Coruña. España ISSN 2386-8333

Deporte, $\quad$ 46, 38-41. Recuperado de:

https://dialnet.unirioja.es/servlet/articulo?codigo $=5764467$

Rojas, M. C., y Wong, Y. D. (2017). Children's active trips to school: a review and analysis. International Journal of Urban Sustainable Development, 9(1), 79-95. DOI: https://doi.org/10.1080/19463138.2016.1264405

Roman-Viñas, B., Zazo, F., Martínez-Martínez, J., Aznar-Laín, S., y Serra-Majem, L. (2018). Results from Spain's 2018 report card on physical activity for children and youth. Journal of Physical Activity and Health, 15(2), 411-412. DOI: https://doi.org/10.1123/jpah.2018-0464

Rothman, L., Macpherson, A. K., Ross, T., y Buliung, R. N. (2018). The decline in active school transportation (AST): a systematic review of the factors related to AST and changes in school transport over time in North America. Preventive Medicine, 111, 314-322. DOI: https://doi.org/10.1016/j.ypmed.2017.11.018

Ruiz-Ariza, A., Pinillos, F. G., Román, P. Á. L., y López, E. J. M. (2015). Niveles de desplazamiento activo en jóvenes de 12-16 años. Un estudio de la provincia de jaén. EmásF: Revista Digital de Educación Física, 34, 71-79. Recuperado de: https://dialnet.unirioja.es/servlet/articulo?codigo $=5386726$

Sanz-Arazuri, E., Ponde-de-León, A., y Fraguela-Vale, R. (2017). Adolescents' active commutes to school and family functioning. Apunts: Educació Física i Esports, 128. DOI: http://doi.org/10.5672/apunts.2014-0983.es.(2017/2).128.02

Smith, M., Hosking, J., Woodward, A., Witten, K., MacMillan, A., Field, A., ... Mackie, H. (2017). Systematic literature review of built environment effects on physical activity and active transport-an update and new findings on health equity. International Journal of Behavioral Nutrition and Physical Activity, 14(1), 158. DOI: https://doi.org/10.1186/s12966-017-0613-9

Stea, T. H., y Torstveit, M. K. (2014). Association of lifestyle habits and academic achievement in Norwegian adolescents: a cross-sectional study. BMC Public Health, 14, 829. DOI: https://doi.org/10.1186/1471-2458-14-829

Te Velde, S. J., Haraldsen, E., Vik, F. N., De Bourdeaudhuij, I., Jan, N., Kovacs, E., ... Bere, E. (2017). Associations of commuting to school and work with demographic variables 


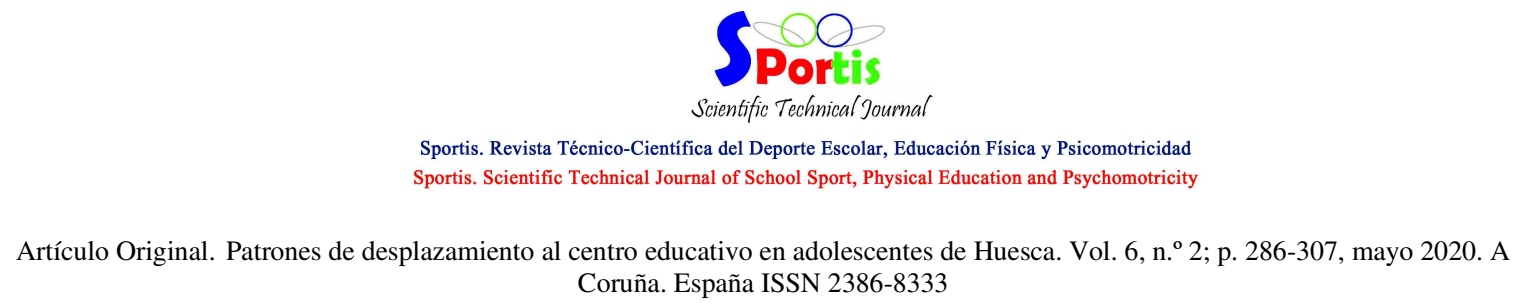

and with weight status in eight European countries: The ENERGY-cross sectional study. Preventive Medicine, 99, 305-312. DOI: https://doi.org/10.1016/j.ypmed.2017.03.005

Uddin, R., Mandic, S., y Khan, A. (2019). Active commuting to and from school among 106,605 adolescents in 27 Asia-Pacific countries. Journal of Transport \& Health, 15, 100637. DOI: https://doi.org/10.1016/j.jth.2019.100637

Villa-González, E., Huertas-Delgado, F. J., Chillón, P., Ramírez-Vélez, R., y Barranco-Ruiz, Y. (2019). Associations between active commuting to school, sleep duration, and breakfast consumption in Ecuadorian young people. BMC Public Health, 19(1), 85. DOI: https://doi.org/10.1186/s12889-019-6434-9

Waygood, E. O. D., Friman, M., Olsson, L. E., y Taniguchi, A. (2017). Transport and child well-being: An integrative review. Travel Behaviour and Society, 9, 32-49. DOI: https://doi.org/10.1016/j.tbs.2017.04.005

World Health Organization (2010). Global recommendations on physical activity for health. World Health Organization, Geneva, Switzerland. Recuperado de: https://www.who.int/dietphysicalactivity/publications/9789241599979/en/

Yang, Y., Ivey, S. S., Levy, M. C., Royne, M. B., y Klesges, L. M. (2016). Active travel to school: findings from the survey of US health behavior in school-aged children, 2009-2010. Journal of School Health, 86(6), 464-471. DOI: https://doi.org/10.1111/josh.12395

Zaragoza, J., Corral, A., Estrada, S., Abós, Á., \& Aibar, A. (2019). Active or passive commuter? Discrepancies in cut-off criteria among adolescents. International Journal of Environmental Research and Public Health, 16(20), 3796. DOI: https://doi.org/10.3390/ijerph16203796 